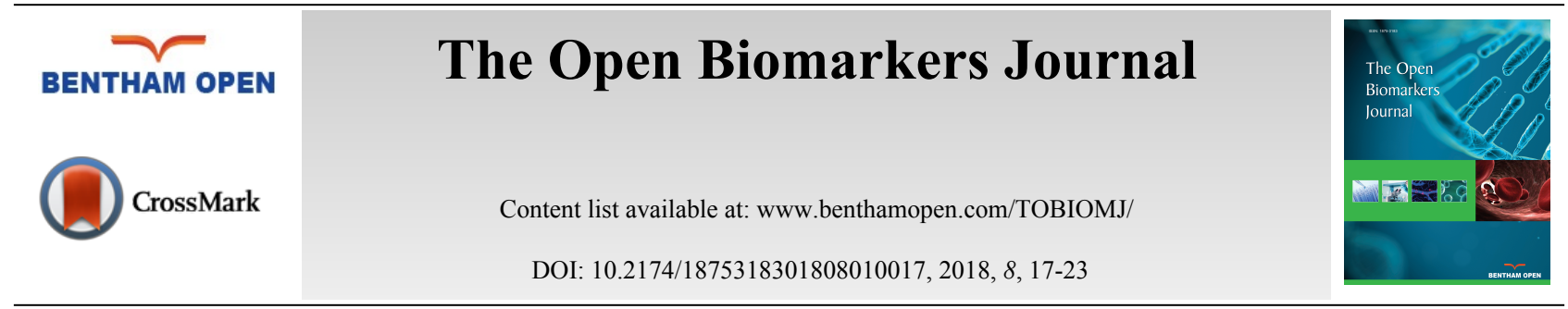

RESEARCH ARTICLE

\title{
Biomarkers of Oxidative Stress and Inflammation in Chagasic Myocardiopathy
}

\author{
Gerrard Gabriela ${ }^{1}$, Martí M. Belén ${ }^{1}$, Diviani Romina ${ }^{1}$, Ceruti M. Jose ${ }^{1}$, Lioi Susana ${ }^{1}$, Beloscar Juan $^{2}$ \\ and D'Arrigo Mabel ${ }^{1, *}$ \\ ${ }^{I}$ Área Química Analítica Clínica, Facultad de Ciencias Bioquímicas y Farmacéuticas, Universidad Nacional de \\ Rosario, Rosario, Santaa Fe, Argentina \\ ${ }^{2}$ Carrera de Cardiología, Facultad de Ciencias Médicas, Universidad Nacional de Rosario, Rosario, Santa Fe, \\ Argentina
}

Received: August 9, 2017

Revised: March 5, 2018

Accepted: October 19, 2018

\section{Abstract:}

\section{Introduction:}

The fact that only part of the population that lives in endemic areas gets Chagas disease and that only some of the patients with chronic infection develop symptoms, supports the importance of investigating the factors of each host in the susceptibility and the development of the disease. Chronic pathological processes and progressive inflammation lead to alterations in the cellular antioxidant status. This imbalance would contribute to the destruction of the parasite and would be related to the cardiac damage observed in patients with chagasic cardiomyopathy.

\section{Objective:}

The objective of the present study was to determine the plasma activity of oxidative stress and inflammatory biomarkers: SOD, CAT, GPx, TBARS and TNF- $\alpha$ in chagasic patients with and without cardiomyopathy and healthy individuals.

Aim:

The aim of the present study is to demonstrate the predisposition to severe forms of chagasic heart disease by quantifying the biomarkers mentioned in blood from the study population.

\section{Results and Conclusion:}

The results show significant differences in the enzymatic activities in the different groups of patients, which would mean at the cellular level, an alteration of the antioxidant capacity. Contrary to what we expected (a depletion of these enzymes), patients show an increase in antioxidant activity, that is, they respond to the generation of free radicals. The same trend is observed in the case of TBARS that are elevated in the case of chagasic patients, indicating a high degree of lipid peroxidation and oxidative damage. Regarding TNF- $\alpha$ levels, we found statistically significant differences, which show an active and chronic inflammatory state in these patients. Although we have found significant differences between the $\mathrm{CN}$ group and the other groups of patients, we should indicate that between the MCC and ECsinMCC groups, the results obtained did not show marked differences. This is important since it has been shown that patients infected with Tc have a marked antioxidant potential and are able to respond to the oxidative stress induced by the parasite, although this would not be decisive in the evolution of the disease.

Keywords: Oxidative stress, Chagas disease, Cardiomyopathy, Inflammation, SOD, CAT, GPx, TNF- $\alpha$.

* Address correspondence to this author at the Área Química Analítica Clínica, Facultad de Ciencias Bioquímicas y Farmacéuticas, Universidad Nacional de Rosario, Rosario, Santaa Fe, Argentina; Tel: +54 341 4804592; E-mails: darrigomabel@yahoo.com.ar 


\section{INTRODUCTION}

There is little knowledge about the underlying mechanisms that cause a chagasic patient to evolve into an irreversible severe condition or to remain in an indeterminate state throughout his life. The presence of Chagas infected individuals, with no obvious cardiac damage, living in endemic areas shows that a proportion of those infected are able to counteract Trypanosoma cruzi (Tc) infection [1]. Chronic pathological processes and progressive inflammation lead to alterations in the cellular antioxidant status. It has been suggested that host genetic factors, environmental factors and Tc variability are the main determinants of the prevalence of Chagas disease and clinical manifestations in humans. Although these factors are probably responsible for the heterogeneity of Chagas disease, there is growing evidence that differential susceptibility in endemic areas may be attributable to host genetic factors [2].

The fact that only a part of the population living in endemic areas is infected and that only a third of people with chronic infection develop symptoms, supports the importance of investigating the factors specific to each host in susceptibility and development of chronic Chagas disease [3].

Oxidative stress is a state of the cell in which the intracellular oxide-reduction homeostasis is altered, that is, the balance between pro-oxidants and antioxidants is affected.This imbalance occurs due to excessive production of Reactive Oxygen Species (ROS) and/or deficiency in antioxidant mechanisms, leading to cellular damage [4]. The ROS mainly comprise superoxide anion $\left(\mathrm{O}_{2}{ }^{-}\right)$, hydrogen peroxide $\left(\mathrm{H}_{2} \mathrm{O}_{2}\right)$, hydroxyl radical $\left(\mathrm{HO}^{*}\right)$ and molecular oxygen $\left(\mathrm{O}_{2}\right)$, while Reactive Nitrogen Species (RNS) include nitric oxide (NO) as well as the peroxynitrite anion $\left(\mathrm{ONOO}^{-}\right)$[5].

Chronic inflammation processes induce oxidative / nitrosative stress and Lipid Peroxidation (LPO), which generate excess of ROS, RNS and reactive aldehydes. It should be noted that it is difficult to determine in vivo free radical levels due to their short half-life, high reactivity and low concentration. Therefore, they are evaluated indirectly by measuring compounds with lower reactivity and longer half-life. Thus, nitrites $\left(\mathrm{NO}_{2}{ }^{-}\right)$are generally measured to evaluate RNS and $\mathrm{O}_{2}{ }_{2}^{-}$and / or $\mathrm{H}_{2} \mathrm{O}_{2}$ to evaluate ROS. The LPO measurement is a good marker to determine oxidative damage of the cell, for which indirect measurements are made, such as the formation of a colored complex of TBA-malondialdehyde (TBARS).

This increase in the production of highly oxidant reactive species and LPO occurs in vivo in Tc infection, as reflected by the increase in plasma $\mathrm{NO}_{2}^{-}$levels as well as malondialdehyde [6]. The generation of ROS could induce tissue damage, similar to that is observed in other pathologies. Unstable radicals attack cellular components causing damage to lipids, proteins and DNA, which can initiate a chain of events that result in cell damage.

In the infected individual, control of the number of parasites would depend primarily on the activation of inducible macrophages Nitric Oxide Synthase (iNOS) and the consequent increase in NO production [7]. The great activation of macrophages and the inflammatory response that contributes to the destruction of the parasite could be related to the damage in myocardium and other tissues observed in the acute phase of the disease [8]. The NO release does not seem to contribute to the formation of inflammatory infiltrates, a fact that also occurs when production is inhibited [9].

Although all living organisms support many endogenous and exogenous factors of oxidative stress, at the same time, they have numerous regulatable systems of antioxidant defenses [10]. In the cell different systems exist that counteract the generation of ROS, those constituted by molecules capable of directly eliminating the ROS, and those based on enzymatic systems that act in cascade to quickly eliminate the $\mathrm{O}_{2}$ - and its metabolites. Antioxidant systems can be classified into two types: primary or enzymatic and secondary or non-enzymatic defense systems.

Among the primary antioxidant systems are those capable of intercepting ROS, preventing the production of new free radicals and consequent damage to cellular structures [11]. In this group are:

- Superoxide dismutase (SOD) that transforms oxygen into hydrogen peroxide. There are several forms of superoxide dismutase, mitochondrial Mn-SOD (SOD2), cytoplasmic Cu / Zn-SOD (SOD1) and extracellular SOD (SOD3). All superoxide dismutases catalyze the conversion of $\mathrm{O}_{2}^{-}$to $\mathrm{O}_{2}$ and $\mathrm{H}_{2} \mathrm{O}_{2}$. Its function is to intercept exogenous $\mathrm{O}_{2}$ avoiding the possible reaction of $\mathrm{NO}$ with $\mathrm{O}_{2}^{-}$, SOD increases the life of NO and decreases the generation of $\mathrm{ONOO}^{-}$, one of the most potent oxidants [12]. SOD, while sequestering superoxide anions, generates $\mathrm{H}_{2} \mathrm{O}_{2}$, another harmful molecule in the cell.

- Glutathione peroxidase ( $\mathrm{GPx}$ ) that converts $\mathrm{H}_{2} \mathrm{O}_{2}$ and lipid peroxides into harmless molecules before they can form free radicals. The biochemical function of glutathione peroxidase is to reduce lipid hydroperoxides to their 
corresponding alcohols and to reduce free hydrogen peroxide to water.

- Catalase (CAT) which is an enzyme found in all known organisms. Catalase is more abundant in the liver, kidney and erythrocytes. In the hepatocyte, catalase is mainly found in peroxisomes, whereas in mature erythrocytes, it is, of course, cytoplasmic. Catalase is an enzyme with a high activity rate and its function is the rapid decomposition of $\mathrm{H}_{2} \mathrm{O}_{2}$ in $\mathrm{O}_{2}$ and $\mathrm{H}_{2} \mathrm{O}$.

Secondary antioxidant systems include examples such as vitamin E, vitamin C, beta-carotene, uric acid, bilirubin, and albumin $[13,14]$.

In relation to $\mathrm{MCC}$, it has been shown that patients who present the chronic form of the disease, have high percentages of inflammation of the myocardium. In this regard, studies on murine models of chronic Chagas disease indicate that inflammatory reactions in the heart are related to an increased production of inflammatory cytokines that induce higher than normal ROS / RNS production [15].

The pathogenesis of MCC is not completely known. Although several antigens and antibodies that cross-react with Tc were identified, the importance of autoimmunity in the pathogenesis is not known [16]. Recent evidence showed that tissue damage is the main action of Tc and the inflammatory response it causes [17, 18].

The growing consensus is that the balance between the persistence of infection and the host immune response is essential for the existence and progression of cardiomyopathy. During the chronic phase, inflammation is the main determinant of progression $[19,20]$.

The inflammation predominates in the cardiac form, with the production of cytokines such as Tumor Necrosis Factor alpha (TNF- $\alpha$ ) and all the cytotoxic mechanisms in which CD $8+$ T cells participate that produce tissue damage and finally severe cardiomyopathy. In the indeterminate chronic form, it predominates the regulatory immunological response, characterized by the production of interleukin 10 and 17 . TNF- $\alpha$ is a proinflammatory cytokine and a potent immunomodulator that is used as a marker of inflammation [21]. It is a polypeptide hormone produced by activated monocytes / macrophages that contribute to the pathogenesis of Chagas disease as it acts as a trypanocidal and tissue injury generator [22]. The production of elevated levels of this cytokine has been demonstrated in experimental animals during the acute phase and its role as an inducer of iNOS influences the control of parasite growth. The iNOS generates $\mathrm{NO}$ that in combination with the $\mathrm{O}_{2}^{-}$from the respiratory outbreak generates $\mathrm{ONOO}^{-}$which is a highly toxic agent for the parasite [23]. TNF- $\alpha$ has been found in the heart of experimentally infected animals as well as in inflammatory infiltrate cells of MCC [24].

\section{OBJECTIVES}

The study aimed to determine in the study population the plasma activity of biomarkers of oxidative stress and inflammation: SOD, CAT, GPx, TBARS and TNF- $\alpha$ in chagasic patients with and without cardiomyopathy and healthy individuals.

This work tries to show the predisposition towards cardiac deterioration, especially the evolution of more severe forms of Chagas' heart disease, quantifying the biomarkers mentioned in blood from the study population.

\section{MATERIALS AND METHODS}

The study population consists of chronic chagasic patients with different degree of cardiac damage and without evidential cardiac damage and control patients without detectable cardiac abnormalities or Chagas' infection.

The patients attended the external office of the Rosario's Centennial Provincial Hospital, all of them received information about the study and signed the consent to participate in the project. Three groups of individuals were analyzed: chagasic patients without MCC (ECsinMCC n: 25), chagasic cardiomyopathy (MCC n: 33) and normal controls (CN n: 55) with similar characteristics, aged between 21 and 70 years, of both sexes, from the city of Rosario and the region (non-endemic area). None of the infected individuals had previously made the study specific antiparasitic treatment (Benznidazole or Nifurtimox). Individuals who presented associated systemic diseases of metabolic, immunological, oncological and / or infectious character, patients on immunomodulatory or immunosuppressive treatments were excluded from the study. The serological diagnosis of Tc infection consisted of at least two positive results from specific serological tests (ELISA, haemagglutination or immunofluorescence). These studies were carried out in the Provincial Hospital's Laboratory. Doctors of the Cardiology Service performed a detailed clinical history of the individuals, which consisted of anamnesis, physical examination, cardiovascular examination, electrocardiogram in 
the 12 conventional leads, chest x-ray and other complementary examinations.

The Ethics Committee of the Faculty of Medical Sciences, National University of Rosario (UNR), Argentina approved the study protocol.

Peripheral venous blood samples were collected, which were collected between 8:00 and 10:00 hs in the morning, the samples were placed in heparinized tubes and dry tubes, and centrifuged at $1500 \mathrm{rpm}$ for 15 minutes to separate the plasma, the erythrocytes and get the serum. The erythrocytes were washed 3 times with saline solution at $4{ }^{\circ} \mathrm{C}$ and the pellets were frozen at $-20^{\circ} \mathrm{C}$ until they were processed.

To analyze the enzymatic activities of SOD, CAT and GPx, erythrocytes were lysed by addition of 4 volumes of distilled water at $4{ }^{\circ} \mathrm{C}$, and diluted to $1 / 500$ with assay buffer. From which, by spectrophotometric methods, SOD (Kits Ransod), CAT (method based on the research of Hugo Aebi), GPx (Kit Ransel), the results were obtained. The measurement of TBARS was performed by spectrophotometric methods developed in our laboratory and the quantification of TNF- $\alpha$ by enzyme immunoassay (HUMAN TNF ELISA -BD OptEIA), the sample analyzed was serum.

To study a possible difference between the groups of patients for each of the variables measured (SOD, CAT, GPx, TBARS, TNF- $\alpha$ ), the unifactorial ANOVA technique was applied. In case of not fulfilling the assumption of normality and / or equality of variances, the Kruskal-Wallis test was applied. Next, multiple comparison tests of Bonferroni or Dunn were applied, as appropriate. In all cases, the level of significance was set at $\mathrm{p}<0.05$.

We must remark that in this research no antioxidant activities were measured in vivo, all the tests carried out have been developed in vitro.

\section{RESULTS}

For SOD, the ANOVA technique was applied. There was a significant difference between the groups $(p=0.0000)$. The Bonferroni's multiple comparisons test showed a significant difference of the control group with all the rest.

For the CAT variable, the ANOVA technique was applied. There was a significant difference between the different groups $(p=0.0014)$. The Bonferroni's multiple comparison tests showed a significant difference between the $\mathrm{CN}$ group with ECsinMCC and with MCC.

For the GPx variable, the Kruskal-Wallis test was applied. This test gave significant $(p=0.00026)$. Dunn's multiple comparisons tests showed a significant difference between $\mathrm{CN}$ and MCC groups $(p=0.0015)$, and between $\mathrm{CN}$ and $\operatorname{ECsinMCC~}(p=0.0008)$.

For TBARS, the Kruskal-Wallis test was applied. This test gave significant $(p=0.0154)$. Dunn's multiple comparisons test showed a significant difference between $\mathrm{CN}$ and MCC $(p=0.0262)$.

For TNF- $\alpha$ Kruskal-Wallis was applied. This test gave significant ( $p=0.001895)$. The Dunn's multiple comparisons tests showed a significant difference between the CN and ECsinMCC groups $(p=0.0425), \mathrm{CN}$ and MCC $(p=0.0050)$. Table 1 contains the results obtained for the measurements of the parameters analyzed.

Table 1. Mean and standard deviations of enzymatic activity of SOD, CAT, GPx, TBARS and TNF- $\alpha$ in patients with Chagas cardiomyopathy (MCC), chagasic patients without MCC (ECsinMCC) and normal controls (CN); and p-value (p).

\begin{tabular}{|c|c|c|c|c|}
\hline & MCC & ECsinMCC & CN & $p$ \\
\hline SOD(USOD/gHb) & $3270 \pm 833$ & $2590 \pm 188$, & $895 \pm 314$ & 0.0000 \\
\hline CAT(K/gHb) & $316 \pm 68$ & $332 \pm 41$ & $185 \pm 28$ & 0.0014 \\
\hline GPx(U/gHb) & $98 \pm 17$ & $102 \pm 20$ & $61 \pm 11$ & 0.00026 \\
\hline TBARS(nmol/ml) & $4.04 \pm 1.82$ & $3.56 \pm 1.22$ & $2.30 \pm 0.62$ & 0.0154 \\
\hline TNF- $\alpha(\mathrm{pg} / \mathrm{ml})$ & $31,3 \pm 16,3$ & $26,6 \pm 12,7$ & $6,4 \pm 4,8$ & 0.001895 \\
\hline
\end{tabular}

Significant differences were observed in the enzymatic activities in the different groups of patients, this would mean at the cellular level, an alteration of the antioxidant capacity. Contrary to what we expected (a depletion of these enzymes), the patients studied respond to the generation of free radicals. We could hypothesize that this alteration of the antioxidant capacity in the final stages of the disease would lead to a depletion of the protective system leading to a fatal outcome in some patients. The same tendency is observed in the case of TBARS that are elevated in the case of chagasic patients indicating a high degree of lipid peroxidation and oxidative damage. Regarding the levels of TNF- $\alpha$ 
we found statistically significant differences which shows an active and chronic inflammatory state in these patients. TNF- $\alpha$ induces iNOS for NO production and curiously also induces Mn-SOD expression but not for other antioxidant enzymes.

\section{CONCLUSION}

The heart contains structural cells, not present in the blood, such as cardiomyocytes and fibroblasts, and these cells could differ in their antioxidant potentials in the groups of cardiac and indeterminate patients. Assuming that the alterations in the antioxidant state in the heart and plasma had the same pathological tendencies, the peripheral blood would be a useful tissue to investigate the impaired mitochondrial function and the antioxidant status in chagasic patients.

Although we have found significant differences between the CN group and the other groups of patients, we should indicate that between the MCC and ECsinMCC groups the results obtained did not show marked differences. Despite these results we can not completely rule out a possible role of antioxidant activity in the development of cardiac versus indeterminate forms of the disease. This is important since it has been demonstrated that patients infected with Tc have a marked antioxidant potential and are able to respond to the oxidative stress induced by the parasite although this would not be determinant in the evolution of the disease, at least in the results obtained by our study group.

The MCC is a complex pathology, since multiple genes participate in the development and evolution of the disease. The information obtained will contribute to broaden our molecular knowledge of the pathophysiology and could lead to better diagnosis and prognosis of this disease. Research should be undertaken to clarify many points concerning organic alterations resulting from parasitism at different stages of their evolution, as in their structural, physiological, biochemical and pathological aspects, to specify the mechanism of these alterations and to provide new bases for diagnosis and treatment.

\section{ETHICS APPROVAL AND CONSENT TO PARTICIPATE}

The Ethics Committee of the Faculty of Medical Sciences, National University of Rosario (UNR), Argentina approved the study protocol.

\section{HUMAN AND ANIMAL RIGHTS}

Animals were not used in this research. All human research procedures followed were in accordance with the ethical standards of the committee responsible for human experimentation (institutional and national), and with the Helsinki Declaration of 1975, as revised in 2013.

\section{CONSENT FOR PUBLICATION}

The patients attended the external office of the Rosario's Centennial Provincial Hospital, all of them received information about the study and signed the consent to participate in the project.

\section{CONFLICT OF INTERESTS}

The author declares no conflict of interest, financial or otherwise.

\section{ACKNOWLEDGEMENTS}

The patients were evaluated by the chair of cardiology, Faculty of Medical Sciences, UNR, located in the Centennial Provincial Hospital, in the city of Rosario, Santa Fe, Argentina. Rosario.

This research is part of the accredited research framework project (MED 266) by the National University of 


\section{REFERENCES}

[1] Correia D, Dias JCP, Prata A. Problems and perspectives for Chagas disease control: In search of a realistic analysis. Rev Soc Bras Med Trop 2008; 41: 193-6.

[http://dx.doi.org/10.1590/S0037-86822008000200012]

[2] De Oliveira B, Filho DW, Pedrosa RC. Oxidative stress in chronic cardiopathy associated with Chagas disease. Int J Cardiol 2007; 116(3): 357-63. [http://dx.doi.org/10.1016/j.ijcard.2006.04.046]

[3] Azevedo EA, Gomes YM, Montenegro SM, Morais CN, Vasconcelos RH. Genetic susceptibility to chronic Chagas disease: An overview of single nucleotide polymorphisms of cytokine genes. Cytokine 2012; 59: 203-8. [http://dx.doi.org/10.1016/j.cyto.2012.04.035]

[4] Garg N, Vyatkina G, Wen JJ. Oxidative damage during chagasic cardiomyopathy development: Role of mitochondrial oxidant release and inefficient antioxidant defense. Free Radic Biol Med 2004; 37(11): 1821-33.

[http://dx.doi.org/10.1016/j.freeradbiomed.2004.08.018]

[5] Machado FS, Martins GA, Silva JS. The role of nitric oxide in the pathogenesis of Chagas disease. Front Biosci 2003; 1(8): $314-25$.

[6] Guedes PM, Gutierrez FR, Mineo TW, Pavanelli WR, Silva JS. The effects of nitric oxide on the immune system during Trypanosoma cruzi infection. Mem Inst Oswaldo Cruz 2009; 104: 236-45. [http://dx.doi.org/10.1590/S0074-02762009000900030]

[7] Turrens JF. Mitochondrial formation of reactive oxygen species. J Physiol 2003; 552(2): 335-44. [http://dx.doi.org/10.1113/jphysiol.2003.049478]

[8] Dutra WO, Rocha MOC, Teixeira MM. The clinical immunology of human Chagas disease. Trends Parasitol 2005; $21: 581-7$. [http://dx.doi.org/10.1016/j.pt.2005.09.007]

[9] Garg N, Manzur RE, Sembaj A, Wen JJ, Yachelini PC. Increased oxidative stress is correlated with mitochondrial dysfunction in chagasic patients. Free Radic Biol Med 2006; 41(2): 270-6. [http://dx.doi.org/10.1016/j.freeradbiomed.2006.04.009]

[10] Garg N, Ghivalli G, Wen J. Oxidative stress in Chagas disease. Interdiscip Perspect Infect Dis 2009; 1: 1-8.

[11] Barnabé C, Briones B, Guégan JF, et al. Severity of chronic Chagas disease is associated with cytokine/antioxidant imbalance in chronically infected individuals. Int J Parasitol 2003; 33(3): 293-9. [http://dx.doi.org/10.1016/S0020-7519(02)00283-7]

[12] Da Cruz IV, Gottlieb MGV, Jobim PF, Müssel DP, Santos AFR, Schwanke CHA. Association among oxidized LDL levels, MnSOD, apolipoprotein E polymorphisms, and cardiovascular risk factors in a Routh Brazilian region population. Genet Mol Res 2005; 4(4): 691-703.

[13] Folzb RJ, Fukaia T, Harrisona DG, Landmessera U. Extracellular superoxide dismutase and cardiovascular disease. Cardiovasc Res 2002; 55: 239-49. [http://dx.doi.org/10.1016/S0008-6363(02)00328-0]

[14] Delgado Roche L, Martínez Sánchez G, Díaz Batista A. (2009) Determination of oxidative stress markers in cardiovascular disease patients, Acta bioquímica clínica latinoamericana;ISSN 0325-2957, versión On-line ISSN 1851-6114

[15] De Oliveira B, Filho DW, Pedrosa RC. Oxidative stress in chronic cardiopathy associated with Chagas disease. Int J Cardiol 2007; 116(3): 357-63.

[http://dx.doi.org/10.1016/j.ijcard.2006.04.046]

[16] Bonney KM, Engman DM. Chagas heart disease pathogenesis: One mechanism or many? Curr Mol Med 2008; 8: 510-8. [http://dx.doi.org/10.2174/156652408785748004]

[17] Tarleton RL. Parasite persistence in the aetiology of Chagas disease. Int J Parasitol 2001; 31: 550-4. [http://dx.doi.org/10.1016/S0020-7519(01)00158-8]

[18] Burgos JM, Diez M, Vigliano C, et al. Molecular identification of Trypanosoma cruzi discrete typing units in end-stage chronic Chagas heart disease and reactivation after heart transplantation Clin Infect Dis 2010; 51: 485-95. [http://dx.doi.org/10.1086/655680]

[19] Dutra WO, Menezes CAS, Magalhães LMD, Gollob KJ. Immunoregulatory networks in human Chagas disease. Parasite Immunol 2014; 36: $377-87$. [http://dx.doi.org/10.1111/pim.12107]

[20] Poveda C, Fresno M, Gironès N, et al. Cytokine profiling in Chagas disease: Towards understanding the association with infecting Trypanosoma cruzi discrete typing units (a BENEFIT TRIAL sub-study) PLoS One 2014; 9: e91154. [http://dx.doi.org/10.1371/journal.pone.0091154]

[21] Vassalli P. The pathophysiology of tumor necrosis factor. Annu Rev Immunol 1992; 10: 411-52. [http://dx.doi.org/10.1146/annurev.iy.10.040192.002211]

[22] Tarleton RL. Tumor necrosis factor [cachectin] production during experimental Chagas' disease. Clin Exp Immunol 1988; 73 : 186-90.

[23] Vespa GNR, Cunha FQ, Silva JS. Nitric oxide is involved in control of Trypanosoma cruzi-induced parasitemia and directly kills the parasite 
in vitro Infect Immun 1994; 62: 5177-82.

[24] Pissetti CW, Correia D, De Oliveira RF, Llaguno MM, Balarin MAS. Genetic and functional role of TNF-alpha in the development trypanosoma cruzi infection PLoS Negl Trop Dis 2011; 5(3): e976.

[http://dx.doi.org/10.1371/journal.pntd.0000976]

\section{(C) 2018 Gabriela et al.}

This is an open access article distributed under the terms of the Creative Commons Attribution 4.0 International Public License (CC-BY 4.0), a copy of which is available at: (https:/creativecommons.org/licenses/by/4.0/legalcode). This license permits unrestricted use, distribution, and reproduction in any medium, provided the original author and source are credited. 